\title{
Preparing Topological States of a Bose-Einstein Condensate
}

\author{
J. E. Williams \& M. J. Holland \\ JILA and Physics Department, University of Colorado, Boulder, CO 80309-0440, USA
}

The burgeoning field of Bose-Einstein condensation in dilute alkali and hydrogen gases has stimulated a great deal of research into the statistical physics of weakly interacting quantum degenerate systems ${ }^{1,2}$. The recent experiments offer the possibility for exploring fundamental properties of low temperature physics in a very controllable and accessible way. One current goal of experimenters in this field is to observe superfluid-like behavior in these trapped Bose gases, analogous to persistent currents in superfluid liquid helium, which flow without observable viscosity, and electric currents in superconductors, which flow without observable resistance. These "super" properties of Bose-condensed systems occur because the macroscopic occupation of a quantized mode provides a stabilizing mechanism that inhibits decay due to thermal relaxation $^{3}$. Here we solve the time-dependent Gross-Pitaevskii equation of motion of the condensate involving two hyperfine atomic states and show how to generate, with extremely high fidelity, topological modes such as vortices that open the door to the study of superfluidity in these new systems. Our approach is inspired by recent experiments investigating a trapped condensate with two strongly coupled internal states $^{4,5}$. We show how the interplay between the internal and motional dynamics can be utilized to prepare the condensate in a variety of interesting configurations.

Since 1995, when Bose-Einstein condensation in a dilute atomic gas was first observed ${ }^{6-8}$, experimenters have sought a method to create a vortex in this system. In a typical experiment, around one million atoms are trapped in a magnetic harmonic potential and cooled below the critical temperature so that condensation occurs into the lowest energy quantized mode. In the usual case, this ground state has no circulation. One proposed scheme for preparing the condensate in a vortex mode $\mathrm{e}^{9-17}$ is to distort the confining potential and mechanically rotate the trap during the cooling process. In this way, the lowest energy mode may be engineered to be circulating about the axis of symmetry. Such an approach is in direct analogy with experiments on vortices in superfluid helium - the asymmetry of the harmonic trap for the atomic gas plays the role of surface roughness of a rotating vessel. Although conceptually this method appears promising for vortex generation in a trapped gas, so far technical difficulties have precluded its successful implementation.
Instead of having the system condense into a vortex mode, an alternative approach is to allow the atoms to condense into the usual ground state and then dynamically generate the vortex from the non-rotating condensate. Several theoretical proposals have been made along these lines which utilize the interaction between the atoms and a specific laser field consisting of a beam of photons with non-zero orbital angular momentum ${ }^{18-20}$.

The method we present here makes use of both of the techniques mentioned-mechanical rotation and the coupling of internal states using an electromagnetic field. It is motivated by the recent observations demonstrating the cyclic twisting and untwisting of the order parameter of a gaseous condensate of rubidium atoms ${ }^{4}$. These recent experiments have suggested the possibility for harnessing the interplay between the internal and motional dynamics in order to fundamentally alter the topological structure of the order parameter of the condensate ${ }^{5}$.

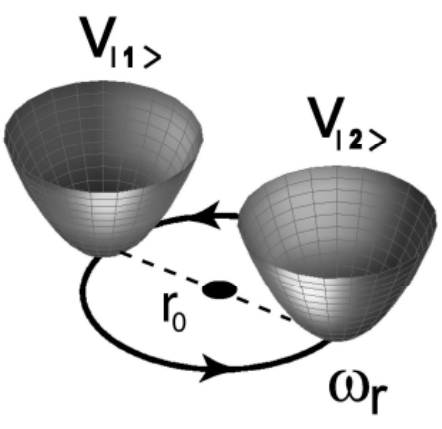

(a)

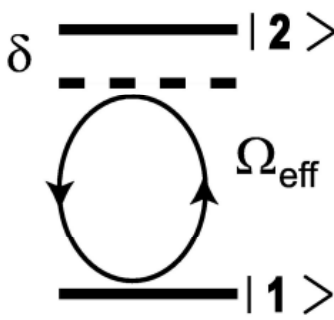

(b)
FIG. 1. Method for creating a vortex. (a) The two traps are rotated in the $x y$-plane about the $z$ axis at a frequency $\omega_{r}$. (b) Transitions are simultaneously driven between the two internal states at the effective Rabi frequency $\Omega_{\text {eff }}$. A vortex mode possessing one unit of angular momentum can be prepared if $\omega_{r} \approx \Omega_{\text {eff }}$.

We treat the internal structure of the condensed atoms as a two-level system as illustrated in Fig. 1. The two states are confined in separate axially symmetric harmonic oscillator potentials with the same trap frequency $\omega_{0}$. As shown in Fig. 1(a), the trap centers are spatially offset by a distance $r_{0}$ and are rotated about the symmetry axis at a frequency of $\omega_{r}$. Simultaneously an electromagnetic field is applied that couples the two internal atomic hyperfine states causing the atoms to coherently cycle between levels as illustrated in Fig. 1(b). There are two parameters that characterize the coupling: the detuning and the power. The detuning $\delta$ denotes the mis- 
match of the frequency of the coupling electromagnetic field to the frequency difference between the two internal atomic states. The power is characterized by the Rabi frequency $\Omega$, which is the rate at which population would oscillate between the two states if $\delta$ were zero. When $\delta$ is larger than $\Omega$, so that the drive is off resonance, the population oscillations have small amplitude and occur at the effective Rabi frequency $\Omega_{\mathrm{eff}}=\sqrt{\Omega^{2}+\delta^{2}}$.

The dynamical evolution of the condensate state $|\psi(t)\rangle$ for a gas of atoms in two different hyperfine states is governed by a nonlinear Schrödinger equation, known as the Gross-Pitaevskii equation, generalized to treat the internal state coupling ${ }^{5}$

$$
\begin{gathered}
i \hbar \frac{\partial}{\partial t}|\psi(t)\rangle=\left[\hat{H}_{0} \otimes \hat{1}+\hat{1} \otimes \frac{\hbar}{2}\left(\Omega \hat{\sigma}_{x}+\delta \hat{\sigma}_{z}\right)\right. \\
\left.+\hat{H}_{1} \otimes \hat{\sigma}_{z}\right]|\psi(t)\rangle,
\end{gathered}
$$

where $\left\{\hat{1}, \hat{\sigma}_{x}, \hat{\sigma}_{y}, \hat{\sigma}_{z}\right\}$ is the set of standard Pauli spin operators and for clarity we have used the tensor product $\otimes$ to explicitly separate the spatial and and internal operators. The free Hamiltonian $\hat{H}_{0}$ describes the motional dynamics of the atoms in a stationary harmonic trap centered on the symmetry axis

$$
\hat{H}_{0}(\boldsymbol{r})=-\frac{\hbar^{2}}{2 m} \nabla^{2}+\frac{1}{2} m \omega_{0}^{2} r^{2}+U_{0} n(\boldsymbol{r}),
$$

where $m$ is the atomic mass. The term that makes the system nonlinear is the mean-field interaction energy, which depends on the local density of condensate atoms $n(\boldsymbol{r})$. The coefficient $U_{0}=4 \pi \hbar^{2} a / m$ is proportional to the scattering length of a binary collision $a$. The effect of displacing the trap centers and rotating them about the symmetry axis is described by

$$
\hat{H}_{1}(\boldsymbol{r}, t)=\kappa\left[f(\boldsymbol{r}) \cos \left(\omega_{r} t\right)+g(\boldsymbol{r}) \sin \left(\omega_{r} t\right)\right],
$$

where $\kappa$ is a coupling coefficient and $f(\boldsymbol{r})$ and $g(\boldsymbol{r})$ are functional prefactors. The explicit form of $\hat{H}_{1}$ determines the symmetry of the quantum state being prepared. To create a vortex state with one unit of angular momentum $\kappa=m \omega_{0}^{2} r_{0}, f(\boldsymbol{r})=x$ and $g(\boldsymbol{r})=y$ in Cartesian coordinates, corresponding to the scheme depicted in Fig. 1(a). More general forms for $f$ and $g$ will be considered later with Eq. (3) then providing the definition of a generalized $\kappa$ and $\omega_{r}$.

Before solving this problem in detail, we first present an intuitive picture of the underlying physics. The vortex state we wish to create has unit circulation corresponding to a macroscopically occupied single particle wave function with quantized angular momentum $\left\langle\hat{L}_{z}\right\rangle / \hbar=1$. In order to see why our scheme couples a non-rotating condensate to this state, consider the frame co-rotating with the trap centers at angular frequency $\omega_{r}$ so that $\hat{H}_{1}$ becomes time-independent. The free Hamiltonian in the co-rotating frame is given by ${ }^{21} \hat{H}_{0}-\omega_{r} \hat{L}_{z}$. The energy of the vortex with one unit of angular momentum is therefore shifted by $\hbar \omega_{r}$ in the rotating frame relative to its value in the lab frame. When this energy shift compensates for both the energy mismatch $\hbar \delta$ of the internal coupling field and the small chemical potential difference between the vortex and non-rotating condensate, resonant transfer of population may take place.

For this picture to be valid, the following inequalities must be satisfied

$$
\omega_{r} \gg \omega_{0}, \quad \delta \gg \Omega, \quad \omega_{r} \approx \Omega_{\mathrm{eff}},
$$

which also imply $\Omega_{\text {eff }} \approx \delta$. The first inequality splits significantly the energies of states of different angular momenta in the rotating frame. This point is crucial for experimental implementation since it allows the vortex to be generated rapidly and is a feature absent from previous dynamic coupling schemes ${ }^{18-20}$. The weak coupling limit given by the second inequality allows the resonance condition $\omega_{r} \approx \Omega_{\text {eff }}$ to select energetically the desired state with high fidelity.

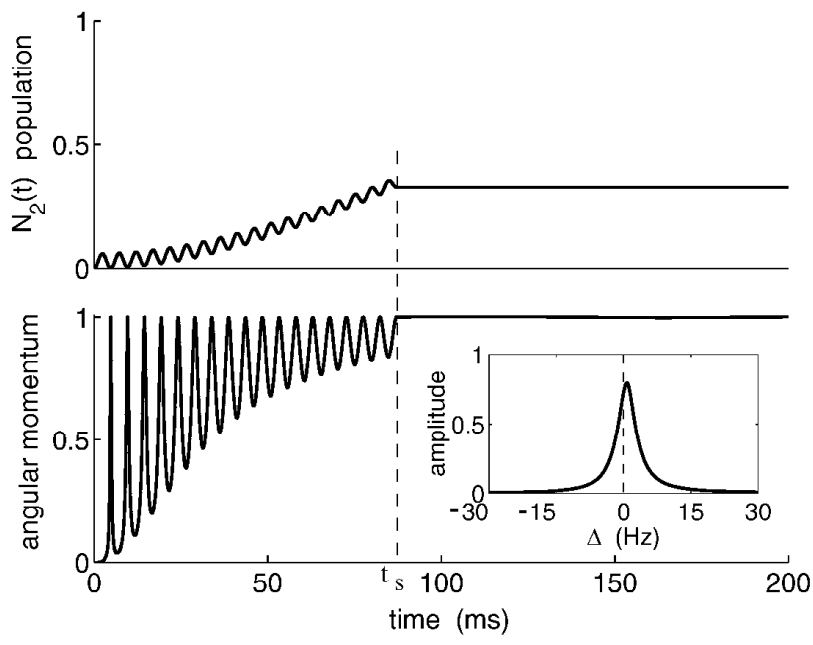

FIG. 2. Dynamical evolution to a vortex. These are results of a numerical integration of Eq. (11), with the condensate initially in the non-rotating ground state and in the internal state $|1\rangle$. The coupling drive is turned on at time $t=0$, and is turned off at time $t=t_{s}$ by setting both $\Omega$ and $r_{0}$ to zero. The top graph shows the fractional population of atoms in the $|2\rangle$ internal state. The small-amplitude rapid oscillations correspond to the cycling between internal levels due to the off-resonant coupling. The gradual rise of this line is due to coupling from the ground state to the vortex mode caused by the drive $\hat{H}_{1}$ in Eq. (3). The bottom graph shows the angular momentum of the $|2\rangle$ state, in units of Planck's constant $\hbar$. The rise and fall of this curve corresponds to a rapid cycling of the $|2\rangle$ atoms between the non-rotating condensate and the vortex. Once during each Rabi cycle, the angular momentum approaches unity and at that time the $|2\rangle$ state wave function approaches a pure vortex mode. The inset shows the maximum amplitude of population transfer to the vortex as a function of the trap rotation frequency $\omega_{r}$, with $\Delta=\Omega_{\text {eff }}-\omega_{r}$. The various parameters used in this calculation are: $\omega_{0}=10 \mathrm{~Hz}, \delta=200 \mathrm{~Hz}, \omega_{r}=205.4 \mathrm{~Hz}$, $N=8 \times 10^{5}$ atoms, $m$ is the mass of the ${ }^{87} \mathrm{Rb}$ atom, $a=5.5$ $\mathrm{nm}^{23}$, and $\Omega=50 \mathrm{~Hz}$ and $r_{0}=1.7 \mu \mathrm{m}$ for $t<t_{s}$. 
Even though we require a large value for $\omega_{r}$, one may not use a simple equivalent time-averaged potential for the effect of rotating the trap centers without including the internal atomic state dynamics in the coarse-graining, which occur on the same time scale.

In order to visualize the system dynamics, we present results from a numerical integration of the GrossPitaevskii equation $^{22}$. Here we treat the system in two dimensions in the $x y$-plane perpendicular to the symmetry axis. In Fig. 2 we plot the fractional population and the angular momentum per atom of the $|2\rangle$ state as a function of time. We initialize the system with all of the atoms in the $|1\rangle$ internal level and in the mean-field ground state of the trap. There are two striking features in Fig. 2. Remarkably, even though we are driving the internal states far from the atomic resonance, a significant fraction of the population gets transferred to the $|2\rangle$ state over a time long compared to the fast Rabi oscillations at $\Omega_{\text {eff }}$. Furthermore, we see that the angular momentum of state $|2\rangle$ oscillates rapidly at the frequency $\Omega_{\text {eff }}$.

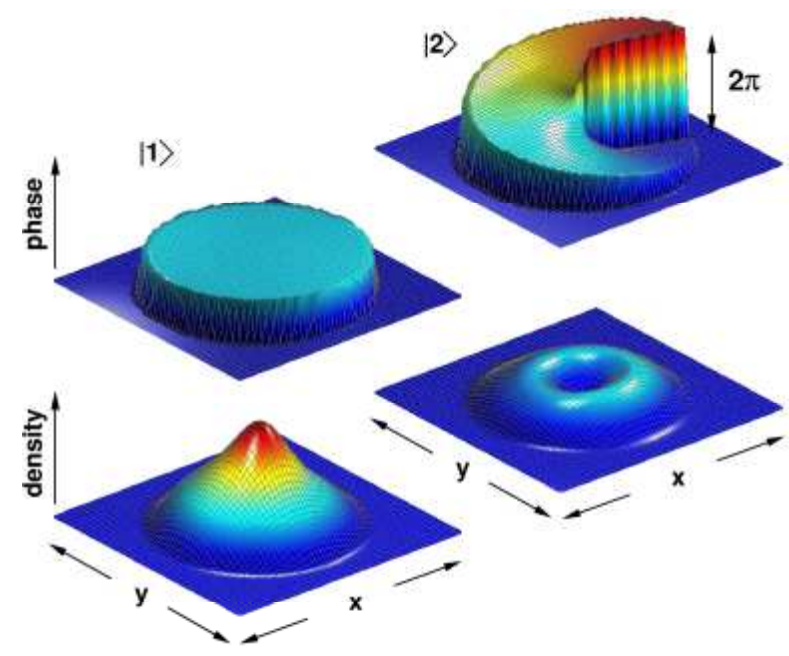

FIG. 3. Unit vortex preparation. Shown are the calculated densities and phases of the two states $|1\rangle$ and $|2\rangle$, at time $t=200 \mathrm{~ms}$ indicated in Fig. 2. At this time, about one third of the atoms are in the $|2\rangle$ state, which is in a pure vortex mode with unit angular momentum. A characteristic feature of a vortex mode is the $2 n \pi$ phase wrap about the core, where $n$ is an integer that is equal to unity in this case. An attractive feature of this preparation scheme is that the $|1\rangle$ atoms residing in the core region provide a natural pinning mechanism for the vortex due to the mean-field repulsion.

Our key idea is that by turning off the coupling at a precise time, $t=t_{s}$, on a given Rabi cycle, the $|2\rangle$ state can be prepared to have unit angular momentum. The maximum possible population transfer to the vortex state using this scheme obeys a Lorentzian response curve as $\omega_{r}$ is varied near $\Omega_{\text {eff }}$ exhibiting a narrow resonance. This is shown in the inset of Fig. 2, where $\Delta=\Omega_{\mathrm{eff}}-\omega_{r}$, and will be discussed in more detail later. In Fig. 3 we show a snapshot of the densities and phases of the two com- ponents at time $t=200 \mathrm{~ms}$. The snapshot illustrates the preparation of a high-quality vortex in state $|2\rangle$, with the 1) state providing a natural "pinning" mechanism that stabilizes the vortex core by providing a repulsive meanfield barrier along the symmetry axis. Consequently the size of the vortex core is determined by the spatial density profile of the non-rotating state, and not by the natural healing length.

An intriguing property of our novel state preparation scheme is that the direction of circulation of the vortex can be opposite to that of the rotating trap centers; changing the sign of the detuning $\delta$ with the direction of rotation of the trap centers fixed causes the vortex to rotate in the opposite direction. This is most easily seen by again considering the frame co-rotating with the potentials at frequency $\omega_{r}$. Vortices with opposite circulations experience opposite energy shifts in transforming to the rotating frame and therefore require opposite signs of detuning in order to achieve resonant coupling.

Although the numerical calculations we present here are full solutions of Eq. (11), an accurate and greatly simplified model may be derived using the separation of time scales given by the inequalities in Eq. (4). This allows us to gain insight into the physical mechanisms giving rise to the behavior of this system. If we coarse-grain over rapidly oscillating terms in order to study the long time scale behavior, we arrive at an approximate solution for the system dynamics, which has the simple form ${ }^{24}$

$$
\begin{aligned}
|\psi(t)\rangle & =\left[a(t) c_{0}(t)\left|\phi_{0}\right\rangle+b(t) c_{n}(t)\left|\phi_{n}\right\rangle\right]|1\rangle \\
& +\left[b(t) c_{0}(t)\left|\phi_{0}\right\rangle+a^{*}(t) c_{n}(t)\left|\phi_{n}\right\rangle\right]|2\rangle .
\end{aligned}
$$

Here $\left|\phi_{0}\right\rangle$ and $\left|\phi_{n}\right\rangle$ are the spatial parts of the state, with $\left|\phi_{0}\right\rangle$ being the ground state and $\left|\phi_{n}\right\rangle$ being the target state - the unit vortex for the case considered in Fig. 3, determined by the self-consistent lowest energy solutions of the two-component Gross-Pitaevskii equation ${ }^{5}$. For given populations of the two atomic states, the energy is minimized using a steepest descents algorithm with the constraint that $\left|\phi_{0}\right\rangle$ is the nodeless ground state and $\left|\phi_{n}\right\rangle$ possesses the symmetry imposed by $\hat{H}_{1}$, which for the unit vortex is a $2 \pi$ topological phase wrap on any closed loop containing the core. The rapidly varying coefficients in Eq. (5) are found to be given by $a(t)=\cos \left(\Omega_{\text {eff }} t / 2\right)-$ $i\left(\delta / \Omega_{\mathrm{eff}}\right) \sin \left(\Omega_{\mathrm{eff}} t / 2\right)$ and $b(t)=-i\left(\Omega / \Omega_{\mathrm{eff}}\right) \sin \left(\Omega_{\mathrm{eff}} t / 2\right)$. The slow coupling of the spatial states is given by $c_{0}$ and $c_{n}$, which satisfy

$$
i \hbar\left(\begin{array}{c}
\dot{c}_{0} \\
\dot{c}_{n}
\end{array}\right)=\frac{1}{2}\left(\begin{array}{cc}
-\left(\epsilon_{n}-\epsilon_{0}-\hbar \Delta\right) & \beta\left\langle\phi_{n}|f+i g| \phi_{0}\right\rangle \\
\beta\left\langle\phi_{0}|f-i g| \phi_{n}\right\rangle & \left(\epsilon_{n}-\epsilon_{0}-\hbar \Delta\right)
\end{array}\right)\left(\begin{array}{c}
c_{0} \\
c_{n}
\end{array}\right) .
$$

Here $\beta=\kappa \Omega /|\delta|$ is the coupling coefficient, $f$ and $g$ are defined in Eq. (3), and $\epsilon_{0}$ and $\epsilon_{n}$ are the energy eigenvalues of $\left|\phi_{0}\right\rangle$ and $\left|\phi_{n}\right\rangle$, respectively. The time scale for state preparation is determined by $\beta$ and the overlap matrix element $\left\langle\phi_{n}|f+i g| \phi_{0}\right\rangle$. These two parameters also determine the width of the response Lorentzian that is shown 
in the inset of Fig. 2 with the maximum occurring not at zero frequency but displaced by an amount according to the splitting between the eigenenergies $\epsilon_{n}-\epsilon_{0}$; typically a small fraction of $\omega_{0}$.

The symmetry of the coupling is determined by $f+i g$, which has the form $x+i y$ as previously stated for the case of the unit vortex. In order to produce a vortex with $n$ units of angular momentum (i.e. $\left.\left\langle\hat{L}_{z}\right\rangle / \hbar=n\right), f+i g$ should take on the form $(x+i y)^{n}$. So for $n=2$, we must construct an $\hat{H}_{1}$ in which $f=x^{2}-y^{2}$ and $g=2 x y$, corresponding to a saddle potential rotating at $\omega_{r} / 2$. In Fig. 4, we illustrate vortex generation with two and three units of angular momentum.

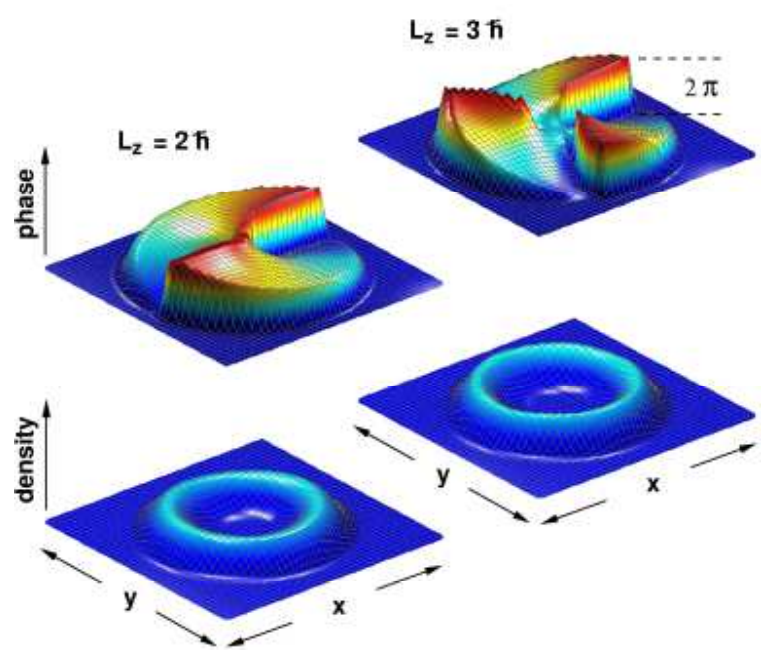

FIG. 4. Double and triple vortex preparation. Shown are the densities and phases modulo $2 \pi$ of the $|2\rangle$ state after a dynamical evolution similar to that shown in Fig. 2, but with different symmetries of the drive $\hat{H}_{1}$. In the case of $\left\langle\hat{L}_{z}\right\rangle / \hbar=2$, $f=x^{2}-y^{2}$ and $g=2 x y$, while for $\left\langle\hat{L}_{z}\right\rangle / \hbar=3, f=x^{3}-3 x y^{2}$ and $g=3 x^{2} y-y^{3}$. In both cases, the system was evolved from the same initial condition as that for the calculation described in Fig. 2, with about one third of the atoms in the $|2\rangle$ state at the time $t_{s}$. The values taken for the various parameters were the same, except for the trap rotation frequency, which was $\omega_{r}=204.3 \mathrm{~Hz}$ for the $\left\langle\hat{L}_{z}\right\rangle / \hbar=2$ case, and $\omega_{r}=200.2$ $\mathrm{Hz}$ for the $\left\langle\hat{L}_{z}\right\rangle / \hbar=3$ case.

The generalization of our scheme for the preparation of macroscopic quantum states of arbitrary symmetry is straightforward. For example, to generate a mode with a dipole symmetry one superimnoses a left and right so that $f=P(x-i y\}$ and $g=0$. A quadrapole symmetry would be generated by $f=\operatorname{Im}\left\{(x+i y)^{2}\right\}$ and $g=0$. The dynamical state preparation of these two examples is illustrated in Fig. 5.

We do not anticipate collisions to play a significant role on the short time scale for vortex generation shown in Fig. 2 and we have implicitly neglected them in our zero temperature theory. However, the system is thermodynamically unstable on long time scales if the rotating drive $\hat{H}_{1}$ is left on, since atoms can access states of higher and higher angular momentum through collisional relaxation. Even after the vortex state has been generated and the drive is turned off, collisions will be important in determining the stability of persistent currents where the topological structure of the two component order parameter is known to be crucial ${ }^{3,12}$.

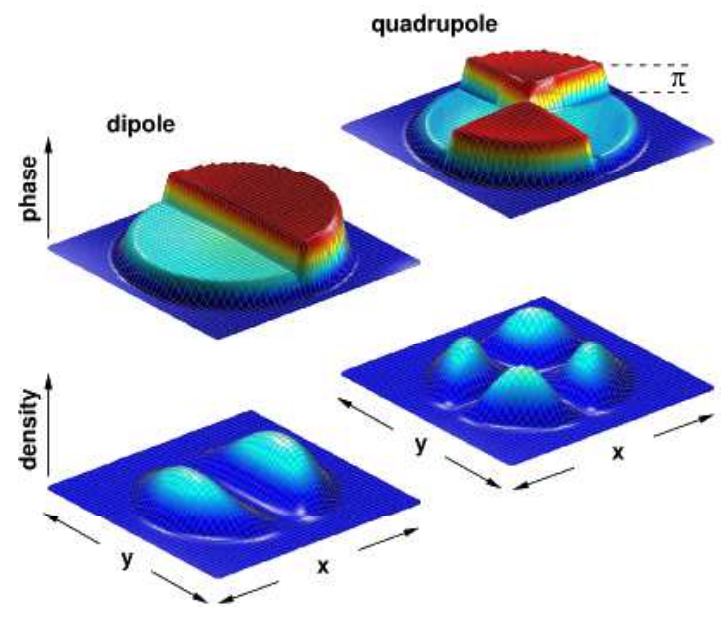

FIG. 5. Dipole and quadrupole preparation. Different symmetries were constructed using specific forms for the drive $\hat{H}_{1}$ in order to prepare the $|2\rangle$ state in non-circulating modes. Instead of having a $2 n \pi$ phase wrap corresponding to a current flowing around a central core, these modes have regions of constant phase separated by a discontinuous jump of $\pi$ where the wave function changes sign. To generate the dipole mode, we used $f=x$ and $g=0$ with $\omega_{r}=205.4 \mathrm{~Hz}$; while for the quadrupole, we used $f=x y$ and $g=0$ with $\omega_{r}=204.3 \mathrm{~Hz}$.

Note added: While this paper was in press, vortices in a two-component Bose-Einstein condensate have been created and observed using this scheme ${ }^{25}$.

1. Cornell, E. A., Ensher, J. R. \& Wieman, C. E. Experiments in Dilute Atomic Bose-Einstein Condensation. Proceedings of the 1998 Enrico Fermi summer school on Bose-Einstein condensation in Varenna, Italy. Preprint http://xxx.lanl.gov condmat/9903109.

2. Ketterle, W., Durfee, D. S. \& Stamper-Kurn, D. M. Making, probing and understanding Bose-Einstein condensates. Proceedings of the 1998 Enrico Fermi summer school on Bose-Einstein condensation in Varenna, Italy, cond-mat/9904034.

3. Leggett, A. Low temperature physics, superconductivity and superfluidity. In The New Physics. Editor Davies, P., Cambridge University Press, London, 268-288 (1989). 
4. Matthews, M. R., Anderson, B. P., Haljan, P. C., Hall, D. S., Williams, J. E., Holland, M. J., Wieman, C. E. \& Cornell, E. A. Watching a superfluid untwist itself: Recurrence of Rabi oscillations in a Bose-Einstein condensate, cond-mat/9906288.

5. Williams, J., Walser, R., Cooper, J., Cornell, E. A. \& Holland, M. Excitation of an antisymmetric collective mode in a strongly coupled two-component Bose-Einstein condensate, cond-mat/9904399.

6. Anderson, M. H., Ensher, J. R., Matthews, M. R., Wieman, C. E. \& Cornell, E. A. Observation of BoseEinstein condensation in a dilute atomic vapor. Science 269, 198-201 (1995).

7. Davis, K. B., Mewes, M.-O., Andrews, M. R., van Druten, N. J., Durfee, D. S., Kurn, D. M. \& Ketterle, W. Bose-Einstein condensation in a gas of sodium atoms. Phys. Rev. Lett. 75, 3969-3973 (1995).

8. Bradley, C. C., Sackett, C. A., Tollet, J. J. \& Hulet, R. G. Evidence of Bose-Einstein Condensation in an Atomic Gas with Attractive Interactions. Phys. Rev. Lett. 75, 1687-1690 (1995); Erratum. Phys. Rev. Lett. 79, 1170-1170 (1997).

9. Butts, D. A. \& Rokhsar, D. S. Predicting signatures of rotating Bose-Einstein condensates. Nature $\mathbf{3 9 7}$, 327-329 (1999).

10. Rokhsar, D. S. Vortex stability and persistent currents in trapped Bose gases. Phys. Rev. Lett. 79, 2164-2167 (1997).

11. Dalfovo, F. \& Stringari, S. Bosons in anisotropic traps: Ground state and vortices. Phys. Rev. A 53, 2477-2485 (1996).

12. Ho, T.-L. \& Shenoy, V. B. Local spin-gauge symmetry of the Bose-Einstein condensates in atomic gases. Phys. Rev. Lett. 77, 2595-2599 (1996).

13. Barenghi, C. F. Vortex waves in a cloud of BoseEinstein-condensed, trapped alkali-metal atoms. Phys. Rev. A 54, 5445-5446 (1996).

14. Fetter, A. L. Vortex stability in a trapped Bose condensate. J. Low Temp. Phys. 113, 189-194 (1998).

15. Feder, D. L., Clark, C. W. \& Schneider, B. I. Vortex stability of interacting Bose-Einstein condensates confined in anisotropic harmonic traps. Phys. Rev. Lett. 82, 4956-4959 (1999).
16. Caradoc-Davies, B. M., Ballagh, R. J. \& Burnett, K. Coherent dynamics of vortex formation in trapped Bose-Einstein condensates. Phys. Rev. Lett. 83, 895-898 (1999).

17. Dodd, R. J., Burnett, K., Edwards, M. \& Clark, C. W. Excitation spectroscopy of vortex states in dilute Bose-Einstein condensed gases. Phys. Rev. A 56, 587-590 (1997).

18. Marzlin, K.-P. \& Zhang, W. Vortex coupler for atomic Bose-Einstein condensates. Phys. Rev. Lett. 79, 4728-4731 (1997).

19. Bolda, E. L. \& Walls, D. F. Creation of vortices in a Bose-Einstein condensate by a Raman technique. Phys. Lett. A 246, 32-36 (1998).

20. Dum, R., Cirac, J. I., Lewenstein, M. \& Zoller, P. Creation of dark solitons and vortices in BoseEinstein condensates. Phys. Rev. Lett. 80, 29722975 (1998).

21. Lifshitz, E. M. \& Pitaevskii, L. P. Statistical Physics Pergamon, Oxford (1980).

22. Holland, M. J., Jin, D. S., Chiofalo, M. L. \& Cooper, J. Emergence of interaction effects in Bose-Einstein condensation. Phys. Rev. Lett. 78, 3801-3805 (1997).

23. Hall, D. S., Matthews, M. R., Ensher, J. R., Wieman, C. E. \& Cornell, E. A. Dynamics of component separation in a binary mixture of Bose-Einstein condensates. Phys. Rev. Lett. 81, 1539-1542 (1998).

24. Williams, J. E. The Preparation of Topological Modes in a Strongly-Coupled Two-Component BoseEinstein Condensate. Ph.D. Thesis, University of Colorado at Boulder (1999).

25. Matthews, M. R., Anderson, B. P., Haljan, P. C., Hall, D. S., Wieman, C. E. \& Cornell, E. A. Vortices in a Bose-Einstein Condensate, cond-mat/9908209.

Acknowledgements. We would like to thank E. A. Cornell, M. R. Matthews, P. C. Haljan, B. P. Anderson, and C. E. Wieman, for extensive discussions on the realization of our scheme. We would also like to thank R. Walser and J. Cooper for helpful comments. This work was supported by the National Science Foundation. 